\title{
Epilepsy and the Ketogenic Diet: Assessment of Ketosis in Children Using Breath Acetone
}

\author{
KATHY MUSA-VELOSO, EXEQUIEL RARAMA, FELIX COMEAU, ROSALIND CURTIS, AND \\ STEPHEN CUNNANE \\ Department of Nutritional Sciences, Faculty of Medicine, University of Toronto, Toronto, Ontario M5S \\ 3E2, Canada [K.M.-V., S.C.]; Alcohol Countermeasure Systems Inc., Mississauga, Ontario L5T 2C6, \\ Canada [E.R., F.C.]; Bloorview MacMillan Children's Centre, Toronto, Ontario M2J 4S9, Canada \\ [R.C.]; Division of Neurology, Toronto Hospital for Sick Children, Toronto, Ontario M5G 1X8, Canada \\ [R.C.]
}

\begin{abstract}
High-fat ketogenic diets increase ketones (acetoacetate, $\beta$-hydroxybutyrate, and acetone) and are used to treat refractory seizures. Although ketosis is an integral aspect of these therapeutic regimens, the direct importance of ketosis to seizure control needs further investigation. An examination of this relationship requires a reliable, minimally invasive measure of ketosis that can be performed frequently. In the present study, we examined the use of breath acetone as a measure of ketosis in children with refractory seizures on a classic ketogenic diet. Results were compared with breath acetone levels in epilepsy and healthy controls. Children on the ketogenic diet had significantly higher fasting breath acetone compared with epilepsy or healthy controls $(2530 \pm 600 \mathrm{nmol} / \mathrm{L}$ versus $19 \pm 9 \mathrm{nmol} / \mathrm{L}$ and $21 \pm 4 \mathrm{nmol} / \mathrm{L}$, respectively; $p<0.05$ ). One hour after consumption of a ketogenic breakfast meal, breath acetone increased significantly in epilepsy and healthy controls $(p<0.05)$, but not in children on a ketogenic diet. Children who were on the
\end{abstract}

\section{ABSTRACT}

ketogenic diet for longer periods of time had a significantly lower fasting breath acetone $\left(R^{2}=0.55, p=0.014\right)$. In one child on the ketogenic diet, breath acetone was determined hourly over a 9-h period, both by gas chromatography and by a prototype handheld breath acetone analyzer. Preliminary results using this handheld breath acetone analyzer are encouraging. Breath acetone may be a useful tool in examining the relationship between ketosis and seizure control and enhancing our understanding of the mechanism of the ketogenic diet. (Pediatr Res 52: 443-448, 2002)

AcAc, acetoacetate

Abbreviations

ACET, acetone

$\boldsymbol{\beta}$-HBA, $\beta$-hydroxybutyrate

KD, ketogenic diet

HHBAA, hand-held breath acetone analyzer
Geylin demonstrated that prolonged fasting could be used to treat intractable epilepsy (1). This led to Wilder's development, in 1921, of a high-fat KD for the treatment of intractable seizures (1). The high-fat KD was intended to mimic the physiologic effects of fasting, while avoiding the obvious limitations of prolonged food deprivation (1). Used successfully for approximately 80 years, the KD continues to be one of the most important therapies for intractable childhood seizures (2).

The efficacy of the KD is clear, but its anticonvulsant mechanism remains an enigma. There are several proposed

Received December 4, 2001; accepted March 7, 2002.

Correspondence: Kathy Musa-Veloso, Department of Nutritional Sciences, University of Toronto, 150 College St., Toronto, Ontario M5S 3E2, Canada; e-mail: kathy.musa@utoronto.ca

Supported by the Dairy Farmers of Canada, Natural Sciences and Engineering Research Council of Canada, the Bloorview Children's Hospital Foundation, the Stanley Thomas Johnson Foundation, and the University of Toronto (Awards Division).

DOI: 10.1203/01.PDR.0000030717.77598.40 mechanisms underlying the antiepilepsy action of the KD (3) and, in each, ketosis is presumed to be important. Although a positive relationship between ketosis and seizure control has been documented in several clinical trials (4-7), results from animal studies have been inconclusive $(8,9)$. In vitro studies assessing the association between ketones and seizure activity have also yielded conflicting results $(10,11)$.

Elucidating whether there is a relationship between ketosis and seizure protection in humans requires a minimally invasive, reliable measure of ketosis that can be performed repeatedly in the home setting with minimum inconvenience to the patient and caregivers. "Ketones" include AcAc, $\beta$-HBA, and ACET. For clinical purposes, ketosis is usually assessed in the blood as $\beta$-HBA, or in the urine as AcAc. Although blood $\beta$-HBA analysis is a reliable measure of systemic ketosis, the frequent assessment of blood ketones in the home setting is neither convenient nor practical. Furthermore, the KD is prescribed predominantly to children, and repeated blood sampling from this population is ethically questionable. Although 
nitroprusside-based urinary dipstick ketone tests are convenient and less invasive, they have been criticized as not accurately reflecting blood ketone levels $(7,12-15)$. A novel measure of ketosis is required that combines the accuracy of the blood test and the convenience of the urine ketone tests.

ACET is a 3-carbon ketone that is produced mainly from the spontaneous decarboxylation of AcAc, and, to a lesser degree, by the enzymatic conversion of AcAc to ACET by AcAc decarboxylase (16). Because ACET is volatile, it can be detected in the breath. In high concentrations, ACET causes the breath to take on a characteristic fruity odor. Dietary manipulations such as fasting and consuming high-fat meals have been shown to cause a significant increase in breath ACET (17-22). Breath ACET has previously been found to be a reliable indicator of blood ketone levels in humans $(19,21,23)$ and in rats (22). Breath ACET is potentially useful as an indicator of ketosis, but it has not yet been evaluated in patients with epilepsy on a KD. Thus, the present study was undertaken to determine breath ACET levels in children with refractory seizures on a KD. The primary objective was to compare these levels to those found in healthy children and epilepsy controls.

\section{METHODS}

Subjects. The study was approved by the Ethical Review Office of the University of Toronto (Toronto, Ontario, Canada), the Bloorview MacMillan Children's Center (Willowdale, Ontario, Canada), and the Toronto Hospital for Sick Children (Toronto, Ontario, Canada). All participants and caregivers were fully informed of the experimental procedures before giving written consent (and assent, where possible). Ten children with refractory seizures who were consuming the classic $\mathrm{KD}$ for at least 1 mo were recruited from the Bloorview MacMillan Children's Center. Ten children on antiepilepsy medication and 13 children with no history of epilepsy were recruited from the Toronto Hospital for Sick Children. None of the subjects were diabetic or on weight-reducing diets.

Study design. Each participant underwent a 12-h overnight fast. Home visits were conducted between 0600 and $1000 \mathrm{~h}$, depending on the child's regular waking and eating schedule, and three fasting breath samples were collected. The participant was then given a ketogenic breakfast meal and, $1 \mathrm{~h}$ later, a second set of triplicate breath samples was collected. The breath samples were analyzed for ACET by gas chromatography. Although it would have been useful to correlate breath acetone with simultaneous urine and blood samples, urine and blood samples were not collected in this particular study.

In one child on the KD, two triplicate sets of breath samples were collected hourly over a 9-h period and analyzed for ACET by both gas chromatography and a HHBAA (Alcohol Countermeasure Systems, Inc., Mississauga, Ontario, Canada). The HHBAA is a battery-operated, rechargeable unit with a light-emitting display. The HHBAA is activated by an on/off switch, and has a warm-up time of approximately $30 \mathrm{~s}$. Once the unit is warmed up, a "beeping" sound is heard, and the message "blo" is displayed, indicating the instrument is ready for a breath sample. The child then provides a breath sample by blowing into a disposable mouthpiece that is inserted directly into the instrument. An ACET reading, in nanomoles per liter, is then displayed. We have calibrated breath ACET measurements by the HHBAA against those analyzed by gas chromatography (data not shown).

Breakfast ketogenic meal. A 4:1 (grams of fat to grams of protein plus carbohydrate) ketogenic breakfast meal was prepared for the children as described by Freeman et al. (24). Foods used in the preparation of the ketogenic breakfast meal depended on the child's preferences. Food choices included bacon, eggs, bologna, salami, cheese, cream cheese, 35\% whipping cream, lettuce, tomatoes, and sausage. Once the child/caregiver decided on the prepared foods, those foods were used to formulate the ketogenic breakfast meal.

Breath sampling and analysis. Most of the children that participated in this study were able to provide breath samples effortlessly. A few children, however, were either too young or too developmentally delayed to understand how to provide a breath sample. In an attempt to interest these children and gain their cooperation, a colorful, noise-producing, inflatable party favor was presented. This party favor became an important educational tool for teaching some of the children how to exhale with moderate force. Once the child was successful at blowing into the party favor, the breath collection bag (EasySampler, Quintron Instrument Company, Milwaukee, WI, U.S.A.) that was to be used for the breath collection process was presented immediately so that the child could adapt the newly learned behavior to the new "toy."

The polyethylene breath collection bag had a volume of 500 $\mathrm{mL}$ and was equipped with a mouthpiece and a side port for a Vacutainer needle holder. The child exhaled through the mouthpiece into the polyethylene bag. Two small perforations in the bag allowed the air to be vented as the subject exhaled. To ensure that the breath sample was alveolar and not "deadspace" air, each breath sample was collected only after the 500 $\mathrm{mL}$ polyethylene bag was fully inflated. Each breath sample was then transferred to a $12-\mathrm{mL}$ evacuated glass tube by momentarily perforating the tube's septum with the Vacutainer needle of the breath bag at the end of the expiration. One milliliter of this breath sample was then stored in a glass, gas-tight syringe equipped with a valve (Hamilton Company, Reno, NV, U.S.A.) and transported to the laboratory for ACET analysis by gas chromatography. All breath samples were analyzed within $4 \mathrm{~h}$ of collection. In the one child from whom breath samples were collected hourly over a $9 \mathrm{~h}$, breath samples were collected and analyzed within $10 \mathrm{~min}$.

Breath ACET concentrations were determined immediately after collection using a gas chromatograph equipped with a flame ionization detector (Hewlett Packard Company, Model 5890, Palo Alto, CA, U.S.A.). The 1.8-m glass column was packed with 80/100 mesh Carbopack (Supelco, Belfonte, PA, U.S.A.). The 3-min chromatographic run was performed at $70^{\circ} \mathrm{C}$, with helium as the carrier gas at a flow rate of 20 $\mathrm{mL} / \mathrm{min}$. The injector temperature was $150^{\circ} \mathrm{C}$ and the detector temperature was $200^{\circ} \mathrm{C}$. At these settings, the retention time of ACET was 2.2 min. All ACET peaks were calibrated using an aqueous ACET solution with a concentration appropriate for the particular group being studied (i.e. either $54.3 \mathrm{nmol} / \mathrm{L}$ or $543 \mathrm{nmol} / \mathrm{L}$ ). A $1-\mu \mathrm{L}$ aqueous sample of the standard was then 
injected into the gas chromatograph. The mean coefficient of variation across triplicate breath and calibrator ACET measurements averaged $6.7 \pm 0.7 \%$ and $1.3 \pm 0.2 \%$, respectively.

A HHBAA was used in one child in whom repeated breath sampling was conducted hourly over a 9-h period. Upon expiring into the HHBAA for a duration of $2.5 \mathrm{~s}$, a digital reading was displayed on the HHBAA that corresponded to the child's breath ACET level. Breath ACET was determined by both gas chromatography and the HHBAA for each sample point. The average of three ACET readings was calculated for each sample point. The mean coefficient of variation across triplicate HHBAA and gas chromatography measures was 5.4 $\pm 1.1 \%$ and $7.5 \pm 1.5 \%$, respectively.

Statistical analyses. Statistical Package for the Social Sciences (SPSS, Chicago, IL, U.S.A.) was used for all statistical analyses, and significance was set at $p<0.05$. A one-way ANOVA was used to determine whether the three groups of children differed in age, percentage ideal body weight (\%IBW), fasting breath ACET, or change in breath ACET after ketogenic meal consumption. The relationship between age and fasting or change in breath ACET was assessed using a linear regression model, with age as the independent variable. To determine the relationship between \%IBW and fasting or change in breath ACET, a nonlinear regression model was used, with \% $\mathrm{IBW}$ as the independent variable. A nonlinear regression model was also used to assess the relationship between fasting breath ACET and length of time on the KD, with length of time on the KD as the independent variable. Within the KD group, the effect of carnitine supplementation on fasting or change in breath ACET was determined using a two-tailed, unpaired $t$ test.

\section{RESULTS}

The three groups of children did not differ significantly in age or \%IBW as calculated from the Anthropometric Standards for the Assessment of Growth and Nutritional Status (25). KD children, epilepsy control children, and healthy children had a mean age of $7.9 \pm 1.3,10.2 \pm 1.5$, and $6.7 \pm 1.1 \mathrm{y}$, respectively, and a mean \%IBW of $91.1 \pm 7.9,105.4 \pm 6.0$, and $120.3 \pm 10.6$, respectively. At the time of the study, all the healthy children were medication-free. None of the children with epilepsy (including those on the KD) were medicationfree (Table 1). The most commonly prescribed anticonvulsant drugs were clobazam (Frisium) and carbamazepine (Tegretol) (Table 2). Carnitine supplements were taken only by KD children who were being treated with valproic acid (Epival) (Table 2).

Table 1. Number of medications taken by ketogenic diet children and epilepsy controls

\begin{tabular}{ccc}
\hline $\begin{array}{c}\text { Number of } \\
\text { medications }\end{array}$ & $\begin{array}{c}\text { Ketogenic diet } \\
\text { children* } \\
(n=10)\end{array}$ & $\begin{array}{c}\text { Epilepsy } \\
\text { controls* }(n=10)\end{array}$ \\
\hline 0 & 0 & 0 \\
1 & 20 & 30 \\
2 & 60 & 50 \\
3 & 20 & 20 \\
\hline
\end{tabular}

* Values are expressed as percentages.
Table 2. Medications consumed by ketogenic diet children and epilepsy controls

\begin{tabular}{lcc}
\hline \multicolumn{1}{c}{ Medication } & $\begin{array}{c}\text { Ketogenic diet } \\
\text { children* } \\
(n=10)\end{array}$ & $\begin{array}{c}\text { Epilepsy } \\
\text { controls* } \\
(n=10)\end{array}$ \\
\hline Acetazolamide (Diamox) & 10 & 10 \\
Clobazam (Frisium) & 60 & 10 \\
Diazepam (Vivol) & 0 & 10 \\
Phenytoin Sodium (Dilantin) & 10 & 0 \\
Ethosuximide (Zarontin) & 10 & 0 \\
Lamotrigine (Lamictal) & 30 & 20 \\
Gabapentin (Neurontin) & 0 & 20 \\
Primidone (Sertan) & 0 & 10 \\
Carbamazepine (Tegratol) & 10 & 70 \\
Topiramate (Topamax) & 10 & 20 \\
Valproic acid (Epival) alone & 0 & 20 \\
Valproic acid (Epival) + carnitine & 50 & 0 \\
Vigabitrin (Sabril) & 10 & 0
\end{tabular}

* Values are expressed as percentages. Most children were on a combination of two anticonvulsant drugs (see Table 1).

Fasting breath ACET ranged from 555 to $6671 \mathrm{nmol} / \mathrm{L}$ in $\mathrm{KD}$ children, from 0 to $98 \mathrm{nmol} / \mathrm{L}$ in epilepsy controls, and from 6 to $61 \mathrm{nmol} / \mathrm{L}$ in healthy controls. KD children had a significantly higher fasting breath ACET compared with epilepsy or healthy controls (Fig. 1). One hour after consumption of the ketogenic meal, breath ACET increased significantly in healthy and epilepsy controls, but not in children on the $\mathrm{KD}$ (Fig. 2A). A difference between groups in the change in breath ACET $1 \mathrm{~h}$ after consumption of the ketogenic breakfast meal was almost statistically significant $(p=0.09$; Fig. $2 B$ ).

There was no significant relationship between age and fasting breath ACET in any of the groups. There was a significant negative relationship between age and change in breath ACET in healthy controls, but not in $\mathrm{KD}$ children and epilepsy controls $\left(R^{2}=0.28, p=0.044\right.$; Fig. 3$)$. There was a significant exponential relationship between \%IBW and fasting breath ACET in healthy controls, but not in KD children and epilepsy controls $\left(R^{2}=0.32, p=0.042\right.$; Fig. 4).

Within the KD group, there was no significant effect of carnitine supplementation on fasting and change in breath ACET. A negative exponential relationship was observed between length of time on the KD and fasting breath ACET $\left(R^{2}\right.$

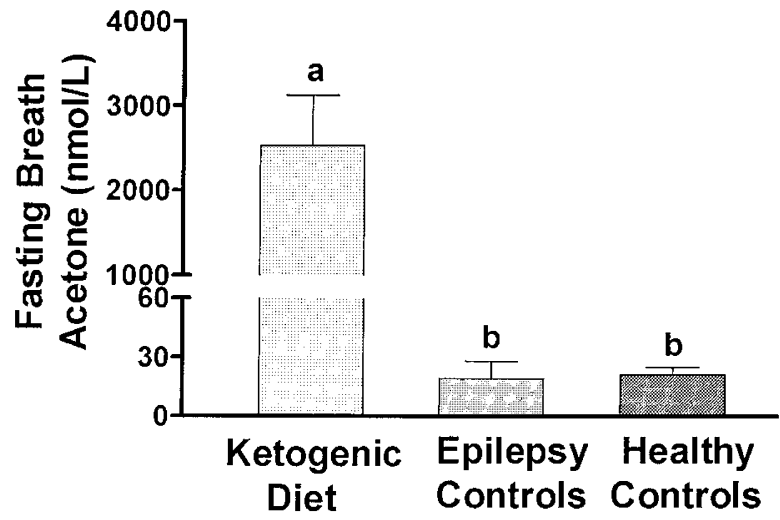

Figure 1. Fasting breath acetone in ketogenic diet children, epilepsy controls, and healthy controls. Bars with different letters are significantly different $(p<$ $0.05)$. 

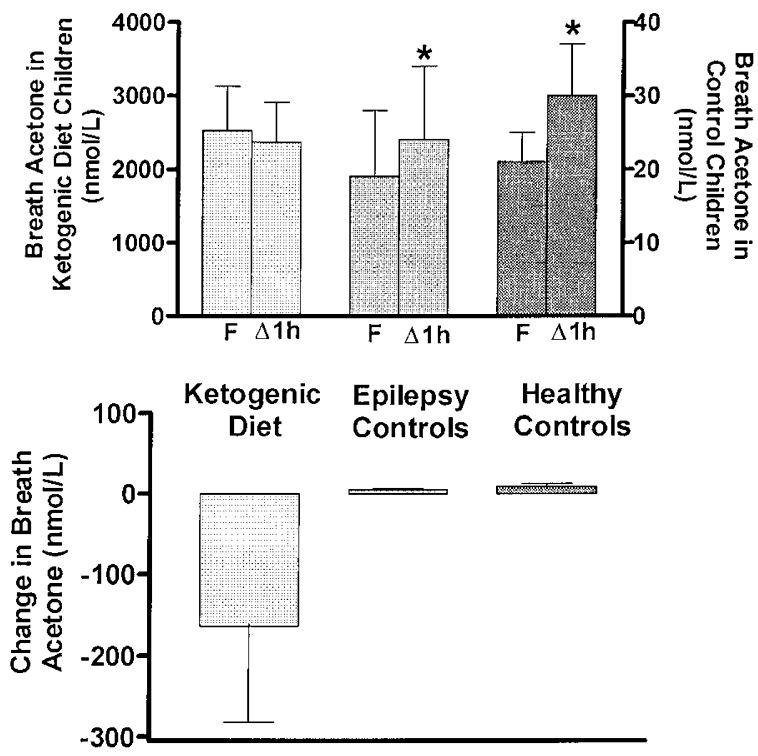

Figure 2. (Top) Breath ACET in KD children, epilepsy controls, and healthy controls. $F$, fasting; $\Delta l h, 1 \mathrm{~h}$ after ketogenic meal consumption. *Significantly greater than at fasting within the same group. (Bottom) Change in breath acetone $1 \mathrm{~h}$ after ketogenic meal consumption in $\mathrm{KD}$ children, epilepsy controls, and healthy controls.

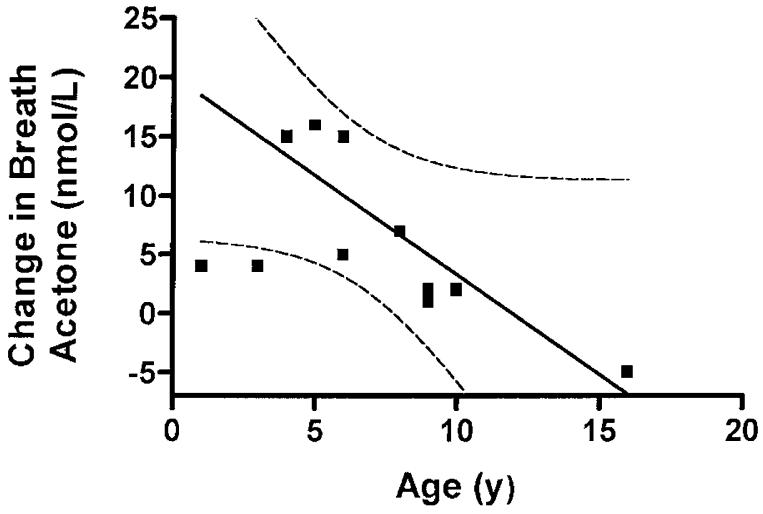

Figure 3. The relationship between age and change in breath ACET in healthy controls. The relationship was best described by a linear model, $\mathrm{Y}=$ $\mathrm{A}+\mathrm{BX}, \mathrm{A}=20.169, \mathrm{~B}=-1.703 ; R^{2}=0.28, p=0.044$.

$=0.55, p=0.014$; Fig. 5). However, there was no significant relationship between length of time on the $\mathrm{KD}$ and change in breath ACET.

Over a 9-h period, breath ACET remained fairly stable in one child with epilepsy on the KD, whether assessed by gas chromatography or the HHBAA (Fig. 6). There was a $1.1 \%$ difference between gas chromatography and HHBAA ACET values (Fig. 6).

\section{DISCUSSION}

In 1927, Talbot et al. (26) examined the biochemical effects of fasting in children with epilepsy and commented on the "marked excretion of ACET bodies shown qualitatively in the breath and urine." Five years later, in a discussion on the mechanism of the $\mathrm{KD}$, Bridge and Iob (27) remarked on the general inverse relationship between the frequency of seizures and the concentration of ketones in the blood, urine, and expired air. Although breath

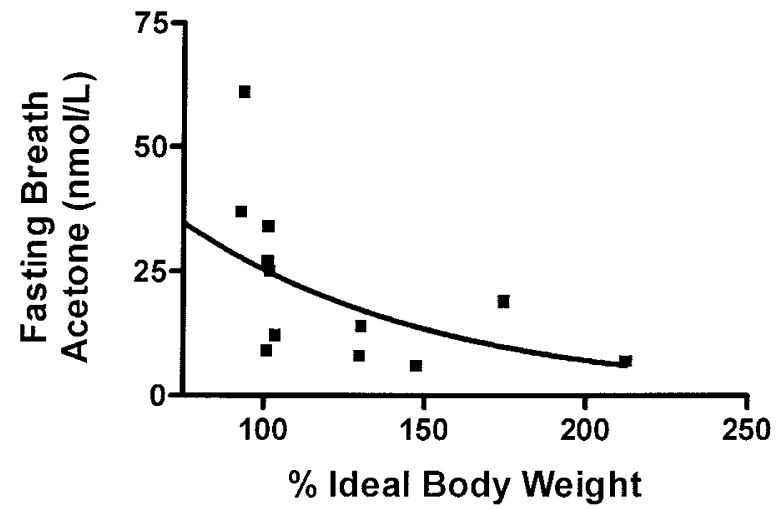

Figure 4. The relationship between percentage of ideal body weight and fasting breath ACET in healthy controls. The relationship was best described by an exponential decay equation, $\mathrm{Y}=\mathrm{A} *\left(\mathrm{e}^{-\mathrm{BX}}\right), \mathrm{A}=60.9834, \mathrm{~B}=0.0106$; $R^{2}=0.32, p=0.042$.

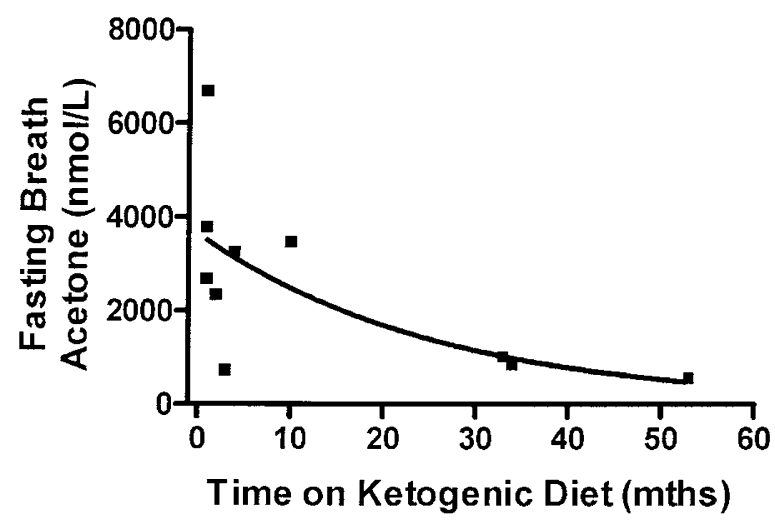

Figure 5. The relationship between time on KD and fasting breath ACET. The relationship was best described by an exponential decay equation, $\mathrm{Y}=$ $\mathrm{A} *\left(\mathrm{e}^{-\mathrm{BX}}\right), \mathrm{A}=3041.48, \mathrm{~B}=0.0332 ; R^{2}=0.55, p=0.014$.

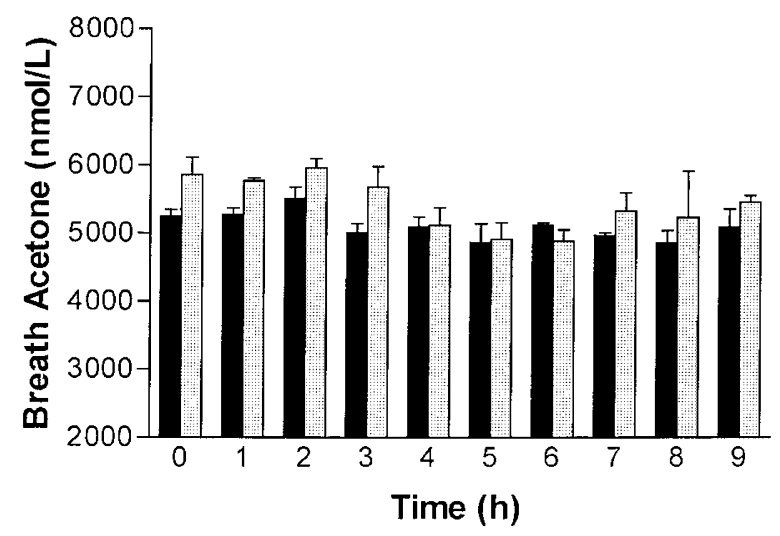

Figure 6. Breath ACET as a function of time in a child on the KD. Solid bar, measured by a HHBAA; dotted bar, measured by gas chromatography.

ACET has been used qualitatively as an indicator of ketosis in children with intractable seizures on a $\mathrm{KD}$, we are unaware of previous reports of quantitative breath ACET measures in this population.

Breath ACET measurements in KD children and epilepsy controls have also not been reported. However, breath ACET in healthy children has been reported to range from 3 to 66 $\mathrm{nmol} / \mathrm{L}$ (28), which is very similar to the range found in healthy children in the present study $(6-61 \mathrm{nmol} / \mathrm{L})$. In chil- 
dren aged 0.1-16 y, Nelson et al. (28) found a significant negative relationship between age and breath ACET. In the current study, a significant relationship between age and breath ACET was not found; however, a significant negative relationship was detected between age and change in breath ACET $1 \mathrm{~h}$ after consumption of the ketogenic breakfast meal. Thus, the younger the child, the greater the postprandial increase in breath ACET. Also, in healthy children, a significant exponential decay association was found between \%IBW and fasting breath ACET, indicating that children heavier than their IBW have a lower fasting breath ACET. This finding is in agreement with earlier observations that heavier people are more resistant to developing ketosis (29).

In the present study, fasting breath ACET was similar in healthy and epilepsy controls, and it increased significantly from fasting $1 \mathrm{~h}$ after consumption of a ketogenic meal. Thus, epilepsy and anticonvulsant drugs do not seem to affect fasting ACET or the production of ACET. Because ACET and the anticonvulsants phenobarbital (Luminal) and felbamate (Felbatol) are metabolized by cytochrome P450 2E1 (30), we postulated that, compared with healthy controls, children with epilepsy on these anticonvulsants would have lower breath ACET levels. Because none of the children in this study were on either of these antiepilepsy drugs, the effect of these drugs on ACET requires further examination.

Compared with healthy and epilepsy controls, children with epilepsy on the KD had a 125 -fold higher fasting breath ACET. One hour after consumption of a ketogenic breakfast meal, breath ACET did not increase significantly from fasting, indicating that children on a KD for at least 1 mo are in stable or maximal ketosis. Although carnitine is essential for mitochondrial uptake (and hence, oxidation) of long-chain fatty acids, KD children who were carnitine-supplemented did not have a significantly higher fasting breath ACET or a significantly greater increase in postprandial breath ACET.

There was a significant inverse association between length of time on the KD and fasting breath ACET. This reduction in ACET over time may have been due to 1) poorer dietary compliance over time; 2) increased tissue uptake of ACET; 3) increased breakdown of ACET via cytochrome P450 2E1; or 4) increased metabolism of ACET, inasmuch as ACET can be used in gluconeogenesis, lipogenesis, and protein synthesis (31). Our present results cannot clearly distinguish between these possibilities. Previous animal studies have shown a tendency for plasma AcAc, plasma $\beta$-HBA, and breath ACET to decrease over time, without an alteration in the formulation/ intake of the $\operatorname{KD}(9,22)$. Thus, in these animal studies, poorer dietary compliance over time did not account for this reduction in ketosis.

There seemed to be little fluctuation in breath ACET over the course of the day. Breath samples were analyzed hourly both by gas chromatography and by the HHBAA. The gas chromatography and HHBAA values were in very close agreement, suggesting that the HHBAA may be an accurate measure of breath ACET. Also, the coefficient of variation across triplicate breath samples was very low, indicating that the HHBAA should be a reliable measure of breath ACET. These preliminary results are promising, and suggest that the
HHBAA may be used to measure breath ACET outside the lab setting. Studies are currently underway to assess the ACET sensitivity range of the HHBAA as well as its sensitivity to other breath components. Our goal is to use the HHBAA to assess the relationship between ketosis and seizure protection in children with intractable seizures on a KD.

We expect that many children on a KD will either be too young or too developmentally/cognitively delayed to use the HHBAA. In collaboration with Alcohol Countermeasure Systems Inc. (Mississauga, Ontario, Canada), we ultimately hope to devise a HHBAA with a sensor that is sufficiently sensitive and specific for ACET that a reading can be generated from a passive breath sample. Because this type of breath sample is not representative of alveolar air, a correction factor would have to be applied. Alternatively, a face mask connected to a ventilation nonrebreathing valve system, such as that described by Nelson et al. (28), could be used to collect breath samples.

Acknowledgments. The authors thank Mary Ann Ryan technical assistance. We also thank Sergei Likhodii for crucial input into the conception that alcohol breathalyzers could be adapted to measuring breath ACET in humans.

\section{REFERENCES}

1. Peterman MG 1924 The ketogenic diet in the treatment of epilepsy: a preliminary report. Am J Dis Child 28:28-33

2. Vining EPG, Freeman JM, Ballaban-Gil K, Camfield CS, Camfield PR, Holmes GL, Shinnar R, Trevathan E, Wheless JW 1988 The Ketogenic Diet Multi-Center Study Group: a multicenter study of the efficacy of the ketogenic diet. Arch Neurol 55:1433-1437

3. Schwartzkroin PA 1999 Mechanisms underlying the anti-epilepsy efficacy of the ketogenic diet. Epilepsy Res 37:171-180

4. McQuarrie I, Keith HM 1927 Relationship of variations in the degree of ketonuria to occurrence of convulsions in epilepsy children on ketogenic diets. Am J Dis Child 34:1013-1029

5. Huttenlocher PR 1976 Ketonemia and seizures: metabolic and anticonvulsant effects of two ketogenic diets in childhood epilepsy. Pediatr Res 10:536-540

6. Whedon B, Sirven JI, O’Dwyer J, Sperling MR, Jefferson 1999 Therapeutic serum B-OH butyrate levels in adult ketogenic diet patients. Epilepsia 40(suppl 7):221(abstr)

7. Gilbert DL, Pyzik PL, Freeman JM 2000 The ketogenic diet: seizure control correlates better with serum $\beta$-hydroxybutyrate than with urine ketones. J Child Neurol 15:787-790

8. Stafstrom CE 1999 Animal models of the ketogenic diet: what have we learned, what can we learn? Epilepsy Res 37:241-259

9. Likhodii SS, Musa K, Mendonca A, Dell C, Burnham WM, Cunnane SC 2000 Dietary fat, ketosis, and seizure resistance in rats on the ketogenic diet. Epilepsia 41:1400-1410

10. Niesen CE, Ge S 1998 The effect of ketone bodies, $\beta$-hydroxybutyrate and acetoacetate, on acute seizure activity in hippocampal CA1 neurons. Epilepsia 39(suppl 6):35(abstr)

11. Thio LL, Wong M, Yamada KA 2000 Ketone bodies do not directly alter excitatory or inhibitory hippocampal synaptic transmission. Neurology 54:325-331

12. Freund G, 1965 The calorie deficiency hypothesis of ketogenesis tested in man. Metabolism 14:985-990

13. Livingston S 1972 Comprehensive Management of Epilepsy in Infancy, Childhood and Adolescence. Charles C Thomas, Illinois, pp 378-405

14. Schwartz RM, Eaton J, Bower BD, Aynsley-Green A 1989 Ketogenic diets in the treatment of epilepsy: short-term clinical effects. Dev Med Child Neurol 31:145-151

15. Laffel L 1999 Ketone bodies: a review of physiology, pathophysiology and application of monitoring to diabetes. Diabetes Metab Res Rev 15:412-426

16. Koorevaar G, Van Stekelenburg GJ 1976 Mammalian acetoacetate decarboxylase activity: its distribution in subfractions of human albumin and occurrence in various tissues of the rat. Clin Chim Acta 71:173-183

17. Freund G, Weinsier RL 1966 Standardized ketosis in man following medium chain triglyceride ingestion. Metabolism 15:980-991

18. Rooth G, Ostenson S 1966 Acetone in alveolar air and the control of diabetes. Lancet 5:1102-1110

19. Tassopoulos CN, Barnett D, Fraser TR 1969 Breath acetone and blood sugar measurements in diabetes. Lancet 1:1282-1286

20. Jones AW 1988 Breath acetone concentrations in fasting male volunteers: further studies and effect of alcohol administration. J Anal Toxicol 12:75-79

21. Musa-Veloso K, Likhodii SS, Cunnane SC 2002 Breath acetone is a reliable indicator of ketosis in adults consuming ketogenic meals. Am J Clin Nutr 76(1):65-70 
22. Likhodii SS, Musa K, Cunnane SC 2002 Breath acetone as a measure of systemic ketosis assessed in a rat model of the ketogenic diet. Clin Chem 48:115-120

23. Owen OE, Caprio S, Reichard Jr GA, Mozzoli MA, Owen RS 1983 Ketosis of starvation: a revisit and new perspectives. Clin Endocrinol Metab 12:359-379

24. Freeman JM, Kelly MT, Freeman JB 1996 The Epilepsy Diet Treatment: An Introduction to the Ketogenic Diet, 2nd Ed. Demos Vermande, New York, pp 131-146

25. Frisancho AR Anthropometric standards for the assessment of growth and nutritional status. In: Shils ME, Olson JA, Shike M, Ross CA (eds) Modern Nutrition in Health and Disease 9th Ed. Lippincott Williams \& Wilkins, Philadelphia, p A-94

26. Talbot FB, Metcalf KM, Moriarty ME 1927 Epilepsy: chemical investigations of rational treatment by production of ketones. Am J Dis Child 33:218-225
27. Bridge EM, Iob LV 1931 The mechanism of the ketogenic diet in epilepsy. Johns Hopkins Med J 48:373-389

28. Nelson N, Lagesson V, Nosratabadi AR, Ludvigsson J, Tagesson C 1998 Exhaled isoprene and acetone in newborn infants and in children with diabetes mellitus. Pediatr Res 44:363-367

29. Kekwick A, Pawan GLS, Chalmers TM 1959 Resistance to ketosis in obese subjects. Lancet 2(2):1157-1159

30. Tanaka E, Terada M, Misawa S 2000 Cytochrome P450 2E1: its clinical and toxicological role. J Clin Pharm Ther 25:167-175

31. Owen OE, Trapp VE, Skutches CL, Mozzoli MA, Hoeldtke RD, Boden G, Reichard GE 1982 Acetone metabolism during diabetic ketoacidosis. Diabetes 31:242-248 